\title{
Abstracts, North American Society for Cardiac Radiology
}

\author{
Presented at the Annual Meeting, San Francisco, April 24 and 25, 1980
}

ETIOLOGIC SIGNIFICANCE OF AN ENLARGED LEFT ATRIAL APPENDAGE IN ADULTS

Michael J. Kelley, Curtis E. Green, Charles B. Higgins University of California, San Diego Medical Center and Veterans Administration Medical Center, 3350 La Jolla Village Dr., San Diego, California 92161

A previous study ${ }^{1}$ has shown that radiographic demonstration of an enlarged left atrial appendage (LAA) in children is a relatively specific indicator of rheumatic mitral valve disease (RMVD). The purpose of the current study was to determine the significance of this finding in adults.

The study population included fifty-one patients whowere divided into two groups: 20 patients with pathologically or clinically proven RMVD and 31 patients with left atrial enlargement (LAE) of a nonrheumatic etiology. The latter group included patients with ischemic papillary muscle dysfunction, mitral valve prolapse, and congestive cardiomyopathy. LAE was determined by chest radiographs and M-mode echocardiograms which were interpreted without knowledge of the clinical history.

Radiographic enlargement of the left atrial appendage (LAA) was present in 18 of 20 rheumatics but in only 1 of 31 nonrheumatics. Presence or absence of the LAA was not related to LA size. In addition, there was no direct relationship between the radiographic size of an enlarged LAA and the radiographic or echocardiographic LA size.

Increased LA pressure alone did not appear to be responsible for LAAE since radiographic findings of pulmonary venous hypertension (PVH) were seen not only in all patients with RMVD but in 23 of the 31 nonrheumatic patients. We postulate that rheumatic inflammation of the LAA allows it to dilate out of proportion to the entire LA, and we conclude that in the adult patient with radiographic findings of PVH, LAAE is a valuable and rather specific radiographic sign of rheumatic mitral valve disease.

1. Kelley, M.J. et al: The significance of left atrial appendage in rheumatic heart disease, Circulation $54: 146,1976$

\section{Three-Dimensional CI Imaging of the Heart}

Erik L. Ritman, Lowell D. Harris, James H. Kinsey, Richard A. Robb, and Barry K. Gilbert

Biodynamics Research Unit, Mayo Clinic, Rochester, Minnesota 55901

A high temporal resolution "volume" imaging system, the Dynamic Spatial Reconstructor (DSR) which was recently installed, has been designed to provide four basic requirements for non-invasive visualization and measurement of anatomic structure and function.

1) A volume is imaged so as to ensure that the region, or section, of interest in an organ is imaged even though the location of this region, or most useful orientation of a section through the organ, is not known until after the image data have been reconstructed and scrutinized. The DSR will image a cylindrically-shaped volume $25 \mathrm{~cm}$ high and up to $33 \mathrm{~cm}$ in diameter.

2) The volume is imaged synchronously so to eliminate the need for gated scanning.
3) Imaging of the volume is in stop-action so as to reduce motion blurring to the point where spatial and density information within the image is not degraded. The DSR will complete a scan within 11 msecs but can be configured to scan for up to 2 or more seconds to achieve higher density and spatial resolution.

4) As movement is a fundamental feature of function of the heart, circulation, and lungs, the volume must be imaged repetitively. The DSR will reinitiate each scan sequence every $1 / 60$ th second.

Because of these characteristics, this scanning system will allow simultaneous evaluation of the dynamic geometry of the heart, of vascular morphology and myocardial perfusion during transient "first pass" angiography.

*The work described in this abstract is supported in part by Grants HL-04664 and RR-00007 from the National Institutes of Health.

Relationship of Coronary Axtery Narrowing and Left Ventricular wall Motion Abnormality: A Quantitative Study.

Harold T. Dodge, David Koh, B. Greg Brown, Douglas K. Stewart, Edward L. Bolson

University of Washington school of Medicine, Division of Cardiology, RG-20, Seattle, WA 98195, USA

In 12 patients with left anterior descending or right coronary artery disease, the relationship between regional wall motion abnormality and degree of coronary narrowing was determined. The patients included three groups: (1) unstable angina without MI, (2) unstable angina with recent subendocardial MI, and (3) previous transmural MI. The majority had significant involvement of a single coronary artery. A computerized method of guantitative analysis of coronary artery dimensions was quantitative ana (Amin) and percent used to determine lesion minimum area (Amin) and percent area stenosis ( 8 ST). Calculated theoretical values inpressure gradient across the lesion at an assumed flow of $1 \mathrm{cc} / \mathrm{sec}$. Using a computerized method, single plane (RaO 30 frames per second) left ventricular (LV) cineangiograms were traced and digitized for each frame in a cardiac cycle. Twenty chords were then constructed at equal intervals perpendicular to the realigned major axis The percent shortening of each chord was compared to the mean of a normal population. Fractional pared to thian (FD) was defined as the distance in standard deviation (FD) was defined as the distance in standar deviations above or below the normal mean. For each patient, an index (SFD) represented the sum of FD's for four adjacent chords with the greatest abso

Diminished segmental wall function as expressed by SFD occurred when Amin was below $0.4 \mathrm{~mm} \mathrm{sq}$. which represented $>92$ stenosis and a computed pressure gradient of $>25$ me wall motion abnormalities were more co mo computed absolute dimensions of the stenotic coronary artery segments and pressure gradient than to percent stenosis. 